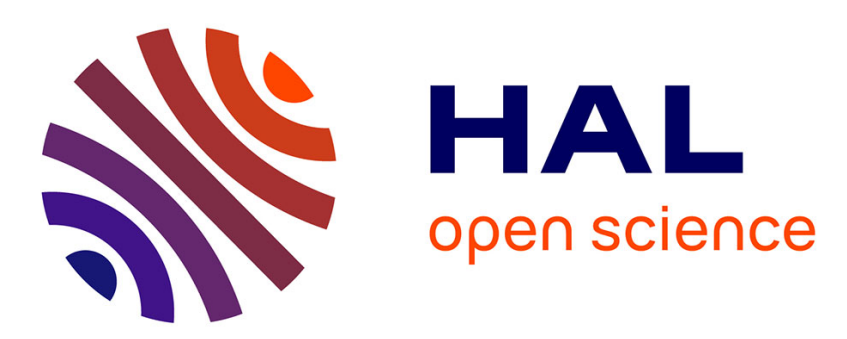

\title{
A numerical tool to compute hybrid Euler-Lagrange compressible models
}

\author{
Jean-Marc Hérard
}

\section{To cite this version:}

Jean-Marc Hérard. A numerical tool to compute hybrid Euler-Lagrange compressible models. 36th AIAA Fluid Dynamics Confernce, Jun 2006, San Fransisco, United States. pp.83-92, 10.2514/6.20062872. hal-01582671

\section{HAL Id: hal-01582671 \\ https://hal.science/hal-01582671}

Submitted on 3 Apr 2018

HAL is a multi-disciplinary open access archive for the deposit and dissemination of scientific research documents, whether they are published or not. The documents may come from teaching and research institutions in France or abroad, or from public or private research centers.
L'archive ouverte pluridisciplinaire HAL, est destinée au dépôt et à la diffusion de documents scientifiques de niveau recherche, publiés ou non, émanant des établissements d'enseignement et de recherche français ou étrangers, des laboratoires publics ou privés. 


\title{
A Numerical Tool To Compute Hybrid Euler-Lagrange Compressible Models
}

\author{
Jean-Marc Hérard * \\ EDF-DRD, 78401 Chatou cedex, France
}

\begin{abstract}
We present in this paper a numerical tool which provides approximations of solutions of hybrid Euler-Lagrange models. The basic underlying ideas of the whole scheme are presented, which rely on the use of relaxation techniques and on a previous investigation of second-moment realisable closures. Numerical tests involving shock and rarefaction waves confirm the suitability of the present approach. The impact of the turbulent noise and the influence of the mesh size are examined.
\end{abstract}

\section{Introduction}

Hybrid PDF methods perfectly compute the Reynolds stress closures and any other correlation, on the basis of a modeled transport equation for the joint PDF ( $\mathrm{se}^{17,18,21,22}$ for instance). One main advantage is that one gets rid of inter-realisability problems, and it also seems to be much more accurate when one aims at computing predictions of turbulent reactive flows. Monte-Carlo methods have become recently quite popular in order to compute approximations of the PDF. Nonetheless, these methods also require the development of accurate and stable enough Finite Volume schemes in order to compute the mean values (i.e. mean density, mean velocity and mean total energy when restricting to compressible flows). The consistent hybrid approach proposed in ${ }^{19,20}$ provides some way to achieve that. It takes advantage of the fact that many upwinding techniques have been developed over the past thirty years in order to compute approximations of Euler equations.

The main objective here is to present another possible way to achieve computations of mean values, assuming a given discrete PDF, or more simply pointwise values of Reynolds stress components. The starting point is the following. When focusing on second-moment closures, it has already been checked (see ${ }^{4,5}$ but also the recent work by Audebert and Coquel ${ }^{1,2}$ ) that standard Euler solvers may fail in some standard situations corresponding to real complex flows. More precisely, the fan of waves in second-moment closures is richer than the one associated with Euler type equations. As a result, wall boundary conditions for instance may generate time-space oscillations, which is due to the fact that some among these waves are actually "ghost waves" for upwinding Euler-type solvers. If one turns then to the work, ${ }^{4,5}$ it occurs that an obvious way to stabilize the whole process simply consists in accounting for the true solution of the exact Riemann problem associated with the governing set of equations for both first and second-order moments. Hence, approximate Riemann solvers may be easily implemented, and these enable to compute approximations of full Reynolds stress models. If one assumes that the prediction of second moments is less trustable than the one asociated with the discrete PDF, this simply suggests to drop second-order moments and to account for moments obtained with help of the PDF instead. This will be very simply achieved with help of a relaxation technique $\left(\right.$ see $^{9}$ and $\left.^{15}\right)$.

Thus, we will focus here on the computation of hybrid models which require computing approximations of mean values of the density $\langle\rho>$, the momentum $(<\rho U>,<\rho V>,<\rho W>)$ and the total energy $<E>$ :

$$
<E>=<\rho e(P, \rho)>+<\rho\left(U^{2}+V^{2}+W^{2}\right)>/ 2+\left(R_{11}+R_{22}+R_{33}\right) / 2
$$

where the Reynolds stress components are denoted $R_{i j}$. The whole must be complemented with some averaged EOS. We consider here an averaged perfect gas equation of state (EOS):

\footnotetext{
*Senior Engineer, Département MFEE, 6 quai Watier, AIAA member
} 


$$
<\rho e(P, \rho)>=<\frac{P}{(\gamma-1)}>=\frac{<P>}{(\gamma-1)}
$$

where $\gamma$ is a given constant greater than one. From now on we will drop all $\tilde{a}$ and $<a>$ notations (used for Favre averaging and Reynolds averaging respectively) and we will use obvious notations.

The paper is organised as follows. We first present the standard approach which is used to predict first and second-order moments in order to account for turbulence within the flow field. We then introduce a sketch of the whole scheme which is grounded on the use of upwinding algorithms and on instantaneous relaxation techniques. Some results will be displayed, which include regular or noisy signals for Reynolds stress closures. We emphasize that our main objective here is to derive a stable tool with respect to mesh refinement and/or noise reduction.

\section{The basic problem}

\section{A. Standard approaches}

One may consider first that the best estimate for the Reynolds stress tensor is given by a Lagrange computation of fluctuations, which provides the full Reynolds stress tensor among other information, at each (space-time) point. In that case, the governing set of Euler equations to be solved in order to get first order moments is:

$$
\begin{array}{r}
\frac{\partial \rho}{\partial t}+\frac{\partial \rho U_{j}}{\partial x_{j}}=0 \\
\frac{\partial \rho U_{i}}{\partial t}+\frac{\partial \rho U_{i} U_{j}}{\partial x_{j}}+\frac{\partial P}{\partial x_{i}}+\frac{\partial R_{i j}^{L a g}}{\partial x_{j}}=0 \\
\frac{\partial E}{\partial t}+\frac{\partial U_{j}(E+P)}{\partial x_{j}}+\frac{\partial U_{i} R_{i j}^{L a g}}{\partial x_{j}}=0
\end{array}
$$

Of course the signal $R_{i j}^{\operatorname{Lag}}(X, t)$ must be provided by some Lagrangian code. In any case, it will fulfill the realisability requirement, that is

$$
0 \leq n_{i} R_{i j}^{L a g} n_{j}
$$

for any vector $n$ in $\mathbb{R}^{3}$.

A very important point to note is that the above mentionned set of PDE is under conservative form, which implies that exact jump conditions are available. In a one dimensional framework, these may be written as:

$$
\begin{array}{r}
-\sigma[\rho]+\left[\rho U_{n}\right]=0 \\
-\sigma\left[\rho U_{n}\right]+\left[\rho U_{n} U_{n}+P\right]=-n_{i}\left[R_{i j}^{L a g}\right] n_{j} \\
-\sigma\left[\rho U_{\tau}\right]+\left[\rho U_{n} U_{\tau}\right]=-\tau_{i}\left[R_{i j}^{L a g}\right] n_{j} \\
-\sigma[E]+\left[U_{n}(E+P)\right]=-\left[U_{i} R_{i j}^{L a g}\right] n_{j}
\end{array}
$$

setting $U_{n}=U_{i} n_{i}, U_{\tau}=U_{i} \tau_{i}$, where we note $n_{j}$ (resp. $\tau_{j}$ ) the components of the unit normal vector aligned with (resp. normal to) the propagation of the plane shock wave, and denoting $\sigma$ the speed of the shock wave.

An alternative classical approach simply consists in a simple Euler prediction of Reynolds stress closures, 
on the basis of objective closures: ${ }^{25}$

$$
\begin{array}{r}
\frac{\partial \rho}{\partial t}+\frac{\partial \rho U_{j}}{\partial x_{j}}=0 \\
\frac{\partial \rho U_{i}}{\partial t}+\frac{\partial \rho U_{i} U_{j}}{\partial x_{j}}+\frac{\partial P}{\partial x_{i}}+\frac{\partial R_{i j}}{\partial x_{j}}=0 \\
\frac{\partial E}{\partial t}+\frac{\partial U_{j}(E+P)}{\partial x_{j}}+\frac{\partial U_{i} R_{i j}}{\partial x_{j}}=0 \\
\frac{\partial R_{i j}}{\partial t}+\frac{\partial U_{k} R_{i j}}{\partial x_{k}}+R_{i k} \frac{\partial U_{j}}{\partial x_{k}}+R_{j k} \frac{\partial U_{i}}{\partial x_{k}}=\frac{\phi_{i j}}{\tau_{1}}
\end{array}
$$

The closure $\phi_{i j}$ may for instance rely on the early proposal by Lumley $\left({ }^{16}\right)$, which takes the form:

$$
\phi_{i j}=\psi_{1}(I, I I, I I I) R_{i j}+\psi_{2}(I, I I, I I I) R_{i j}^{2} / I+\psi_{3}(I, I I, I I I) R_{i j}^{3} / I^{2}
$$

where $I=\operatorname{trace}(R)=R_{l l}, I I=\operatorname{trace}\left(R^{2}\right)=R_{l k} R_{k l}$, and $: I I I=\operatorname{trace}\left(R^{3}\right)=R_{l k} R_{k m} R_{m l}$. $\tau_{1}$ stands for the time scale for the turbulent dissipation. The $\psi_{k}$ are non dimensional bounded functions of $I, I I, I I I$.

It should of course agree with the constraint :

$$
\phi_{l l} \leq 0
$$

In that case, it is known that the regular solutions of the system ((11)-(14)) comply with the over-realisability constraint (see, $\left.{ }^{23},{ }^{12},{ }^{13},{ }^{514}\right)$. This is still true in the limit of weak enough shocks $\left({ }^{4}\right)$. For further details, one may also refer to the recent work by Audebert $(1,2)$.

\section{B. An alternative approach}

We now propose a way to account for both approaches. This simply consists in computing approximations of turbulent fluctuations, which enable to compute $R_{i j}^{L a g}$, and meanwhile to compute approximations of solutions of the system:

$$
\begin{array}{r}
\frac{\partial \rho}{\partial t}+\frac{\partial\left(\rho U_{j}\right)}{\partial x_{j}}=0 \\
\frac{\partial \rho U_{i}}{\partial t}+\frac{\partial\left(\rho U_{i} U_{j}\right)}{\partial x_{j}}+\frac{\partial P}{\partial x_{i}}+\frac{\partial R_{i j}}{\partial x_{j}}=0 \\
\frac{\partial E}{\partial t}+\frac{\partial\left(U_{j}(E+P)\right)}{\partial x_{j}}+\frac{\partial\left(U_{i} R_{i j}\right)}{\partial x_{j}}=0 \\
\frac{\partial R_{i j}}{\partial t}+\frac{\partial\left(U_{k} R_{i j}\right)}{\partial x_{k}}+R_{i k} \frac{\partial U_{j}}{\partial x_{k}}+R_{j k} \frac{\partial U_{i}}{\partial x_{k}}=\mu_{1} \frac{\phi_{i j}}{\tau_{1}}+\mu_{2} M \frac{\left(R_{i k}-R_{i k}^{L a g}\right) R_{k j}+\left(R_{j k}-R_{j k}^{L a g}\right) R_{k i}}{\tau_{2} I}
\end{array}
$$

where :

$$
M=\frac{\left(R_{k l}^{L a g} R_{l k}-R_{k l} R_{l k}\right)}{\left(\left|R_{k l}^{L a g} R_{l k}\right|+R_{k l} R_{l k}\right)}
$$

and $\mu_{k}$ stands for some value in $[0,1]$, and $\tau_{k}$ designs some positive time scale for a return to equilibrium.

The couple $\left(\mu_{1}, \mu_{2}\right)=(1,0)$ has been already investigated (see above). Focus will be given here on $\left(\mu_{1}, \mu_{2}\right)=(0,1)$ or any couple $\left(\mu_{1}, \mu_{2}\right)$ such that $\mu_{1} \mu_{2}$ is non zero.

\section{An overall entropy inequality}

We now introduce the specific entropy $s$ in agreement with :

$$
\rho \frac{\partial s(P, \rho)}{\partial \rho}+\gamma P \frac{\partial s(P, \rho)}{\partial P}=0
$$


and set :

$$
\begin{array}{r}
\eta=\rho \log (s) \\
F_{\eta}^{i}=\rho U_{i} \log (s)
\end{array}
$$

The following holds for regular solutions of ((17)-(20)) :

$$
\frac{\partial \eta}{\partial t}+\frac{\partial F_{\eta}^{i}}{\partial x_{i}}=\frac{\frac{\partial \log (s(P, \rho))}{\partial P}}{\frac{\partial e(P, \rho)}{\partial P}}\left(\mu_{1} \frac{\phi_{k k}}{2 \tau_{1}}-\mu_{2} \frac{\left(R_{k l}^{L a g} R_{l k}-R_{k l} R_{l k}\right)^{2}}{\left(\left|R_{k l}^{L a g} R_{l k}\right|+R_{k l} R_{l k}\right) \tau_{2} I}\right)
$$

Since $\phi_{k k}<0$, the whole provides the entropy inequality :

$$
\frac{\partial \eta}{\partial t}+\frac{\partial F_{\eta}^{i}}{\partial x_{i}} \leq 0
$$

for all regular solutions of ((17)-(20)), using standard equations of state.

This entropy inequality is compulsory if one aims at selecting physically relevant solutions through shock waves.

\section{The coupling scheme}

We restrict now to statistical two-dimensional turbulence in the $(x, y)$ plane, thus setting : $R_{13}=R_{23}=0$, and of course $W=0$, and $\frac{\partial \phi}{\partial z}=0$, whatever $\phi$ is. The EOS thus reads : $E=\rho e(P, \rho)+\rho\left(U^{2}+V^{2}\right) / 2+$ $\left(R_{11}+R_{22}+R_{33}\right) / 2$, where:

$$
(\gamma-1) \rho e(P, \rho)=P
$$

The scheme requires computing solutions of an evolution step which is followed by a relaxation step, both of which are in agreement with the overall entropy inequality.

\section{A. Evolution step:}

The evolution step corresponds to the simulation of $((17),(20))$ while setting $\mu_{2}=0$, using the closures (1) and (2).

The structure of the evolution step guarantees that both regular solutions and shock solutions will be endowed with the over-realisability property. The last Reynolds stress component $R_{33}$ is fully uncoupled.

\section{B. Relaxation step:}

The relaxation step consists in computing approximations of the following:

$$
\begin{array}{r}
\frac{\partial \rho}{\partial t}=\frac{\partial \rho U}{\partial t}=\frac{\partial \rho V}{\partial t}=\frac{\partial E}{\partial t}=0 \\
\frac{\partial R_{i j}}{\partial t}=\mu_{2} M \frac{\left(R_{i k}-R_{i k}^{L a g}\right) R_{k j}+\left(R_{j k}-R_{j k}^{L a g}\right) R_{k i}}{\tau_{2} I}
\end{array}
$$

In the limit of a vanishing time scale $\tau_{2}=0$, the relaxation step simply ensures that:

$$
R_{i j}=R_{i j}^{L a g}
$$

at any time for every point. 


\section{Numerical approach and sample results}

\section{A. Numerical schemes}

The overall algorithm first requires computing approximations of the evolution step. This is achieved by integrating governing equations of the evolution step over control volumes, and using approximate Riemann solvers to provide intermediate states through interfaces between cells. Structured or unstructured meshes may be used. The approximate Riemann solver requires some classical limitation on the time step to comply with the CFL condition. In pratice, we use $C F L=0.5$.

The Riemann solver is obtained while neglecting transverse derivatives. This actually makes sense since the whole system is invariant under frame rotation. Thus, if $n$ designs the direction normal to some interface between two cells, one needs to construct the solution of the one-dimensional Riemann problem associated with:

$$
\begin{array}{r}
\frac{\partial \rho}{\partial t}+\frac{\partial \rho U_{n}}{\partial n}=0 \\
\frac{\partial \rho U_{n}}{\partial t}+\frac{\partial \rho U_{n}^{2}+P+R_{n n}}{\partial n}=0 \\
\frac{\partial \rho U_{\tau}}{\partial t}+\frac{\partial \rho U_{n} U_{\tau}+R_{n \tau}}{\partial n}=0 \\
\frac{\partial E}{\partial t}+\frac{\partial U_{n}(E+P)+U_{n} R_{n n}+U_{\tau} R_{n \tau}}{\partial n}=0 \\
\frac{\partial R_{n n}}{\partial t}+\frac{\partial\left(U_{n} R_{n n}\right)}{\partial n}+2 R_{n n} \frac{\partial U_{n}}{\partial n}=\mu_{1} \frac{\phi_{n n}}{\tau_{1}} \\
\frac{\partial R_{\tau \tau}}{\partial t}+\frac{\partial\left(U_{n} R_{\tau \tau}\right)}{\partial n}+2 R_{n \tau} \frac{\partial U_{\tau}}{\partial n}=\mu_{1} \frac{\phi_{\tau \tau}}{\tau_{1}} \\
\frac{\partial\left(U_{n} R_{n \tau}\right)}{\partial n}+R_{n \tau} \frac{\partial U_{n}}{\partial n}+R_{n n} \frac{\partial U_{\tau}}{\partial n}=\mu_{1} \frac{\phi_{n \tau}}{\tau_{1}} \\
\frac{\partial R_{33}}{\partial t}+\frac{\partial\left(U_{n} R_{33}\right)}{\partial n}=\mu_{1} \frac{\phi_{33}}{\tau_{1}}
\end{array}
$$

together with :

$$
E=\frac{P}{\gamma-1}+\rho\left(U_{n}^{2}+U_{\tau}^{2}\right) / 2+\left(R_{n n}+R_{\tau \tau}+R_{33}\right) / 2
$$

If the turbulence vanishes, the governing equations obviously coincide with Euler equations.

The most tricky part is connected with the approximations of solutions of the evolution step. We recall very briefly that the homogeneous left hand side is an hyperbolic system, whose eigenvalues are :

$$
\begin{aligned}
\lambda_{1} & =U_{n}-c_{1} \\
\lambda_{2} & =U_{n}-c_{2} \\
\lambda_{3}=\lambda_{4}=\lambda_{5} & =\lambda_{6}=U_{n} \\
\lambda_{7} & =U_{n}+c_{2} \\
\lambda_{8} & =U_{n}+c_{1}
\end{aligned}
$$

We note here as usual :

$$
\begin{array}{r}
c_{1}=\left(\frac{\gamma P+3 R_{n n}}{\rho}\right)^{1 / 2} \\
c_{2}=\left(\frac{R_{n n}}{\rho}\right)^{1 / 2}
\end{array}
$$

There are only two Genuinely Non Linear fields which are connected with $\lambda_{1}$ and $\lambda_{8}$. Other fields are Linearly Degenerated. If one assumes that the field only contains weak enough shocks in genuinely non linear fields, the one dimensional Riemann problem admits a unique entropy consistent solution, as detailed in. ${ }^{4}$ 
For practical reasons, we need to extend the notion of upwinding schemes developed within the conservative framework to the frame of non-conservative schemes. For all considerations refereing to the latter item, we thus refer to ${ }^{6}$ for instance, and to, ${ }^{4,5}$ which provide details of the computation of Reynolds stress models in an unsteady framework, and also to,${ }^{3} .{ }^{1}$ For sake of simplicity, the approximate Godunov scheme introduced in ${ }^{7}$ has been used here to predict solutions in the evolution step. We also emphasize that there are many simple numerical schemes which provide approximations that comply with the over-realisability concept, when one incorporates the source terms on the RHS of $((31),(37))$.

\section{B. Numerical results}

The results presented herein correspond to the simulation of a Riemann problem with discontinuous initial data for the mean density, the mean velocity and the mean pressure, on each side of $x=0.5$.

$$
\begin{array}{r}
\rho_{L}=1 . \quad \text { and } \rho_{R}=0.25 \\
U_{L}=0 . \text { and } U_{R}=0 . \\
V_{L}=0 . \text { and } V_{R}=0 . \\
P_{L}=10^{5} \text { and } P_{R}=2.10^{4} \\
R_{11, L}=R_{22, L}=R_{33, L}=10^{3} \quad \text { and } \quad R_{11, R}=R_{22, R}=R_{33, R}=10^{3} \\
R_{12, L}=5.10^{2} \text { and } R_{12, R}=5.10^{2}
\end{array}
$$

The $R_{i j}$ components are given as:

$$
R_{i j}(x, t)=R_{i j}^{0}\left(1+R M S(x) \sin \left(\omega_{1} x-\omega_{2} t\right)+R M S 1(x) \operatorname{rand}(x, t)\right)
$$

The constant $\gamma$ is set to $7 / 5$.

The first test case corresponds to the following data: $\omega_{1}=1000, \omega_{2}=0, \operatorname{RMS}(x)=0.1, \operatorname{RMS} 1(x)=0$., for $x \in[0.25,0.75]$, and $R M S(x)=R M S 1(x)=0$ otherwise. Meshes contain 100 cells up to 100000 cells. Results displayed here have been obtained using 5000 cells. A shock wave propagates to the right, and one recognizes the classical profile for the mean density (figure 1), and for the mean axial velocity (figure 2). Due to gradients of $R_{i j}$ components, the transverse velocity (figure 2), which has been set to zero everywhere in the computational domain, develops now. Obviously results are much depending on the mesh size, when one focuses on rather coarse meshes (see figure 3, which displays mean pressure results when using 500 cells and 10000 cells). This is expected since the frequency of the signal $R_{i j}$ is rather high here. Once the mesh size is in agreement with the Shannon frequency, the dependence becomes much weaker.

Similar results which have been obtained using "random" Reynolds stress data are displayed on figures $(4,5,6)$, while setting: $R M S(x)=0.1, R M S 1(x)=0.02$, for $x \in[0.25,0.75]$, and $R M S(x)=R M S 1(x)=0$ otherwise. .

\section{Conclusion}

This new numerical tool takes advantage of some recent work in order to compute hybrid Euler-Lagrange compressible models. This work is still in progress. Up to now, we have been focusing on the "classical" effect of mesh refinement, whenever one considers regular Reynolds stress profiles, or alternatively noisy signals. Actually, many basic computations seem to confirm that the whole scheme is still stable when using random data for $R_{i j}$ components. We are also currently investigating the impact of the evolution step on computational results. The latter is of major concern. Preliminary results show that computational results are independent of the choice of the evolution step, which is indeed crucial, but still requires further investigation for confirmation.

A companion work ${ }^{8}$ is devoted to a similar approach in order to compute hybrid Euler-Lagrange models for the prediction of two-phase flows. 


\section{Figures}

Riemann problem using imposed Reynolds stress profile $(\mathrm{CFL}=0.5)$

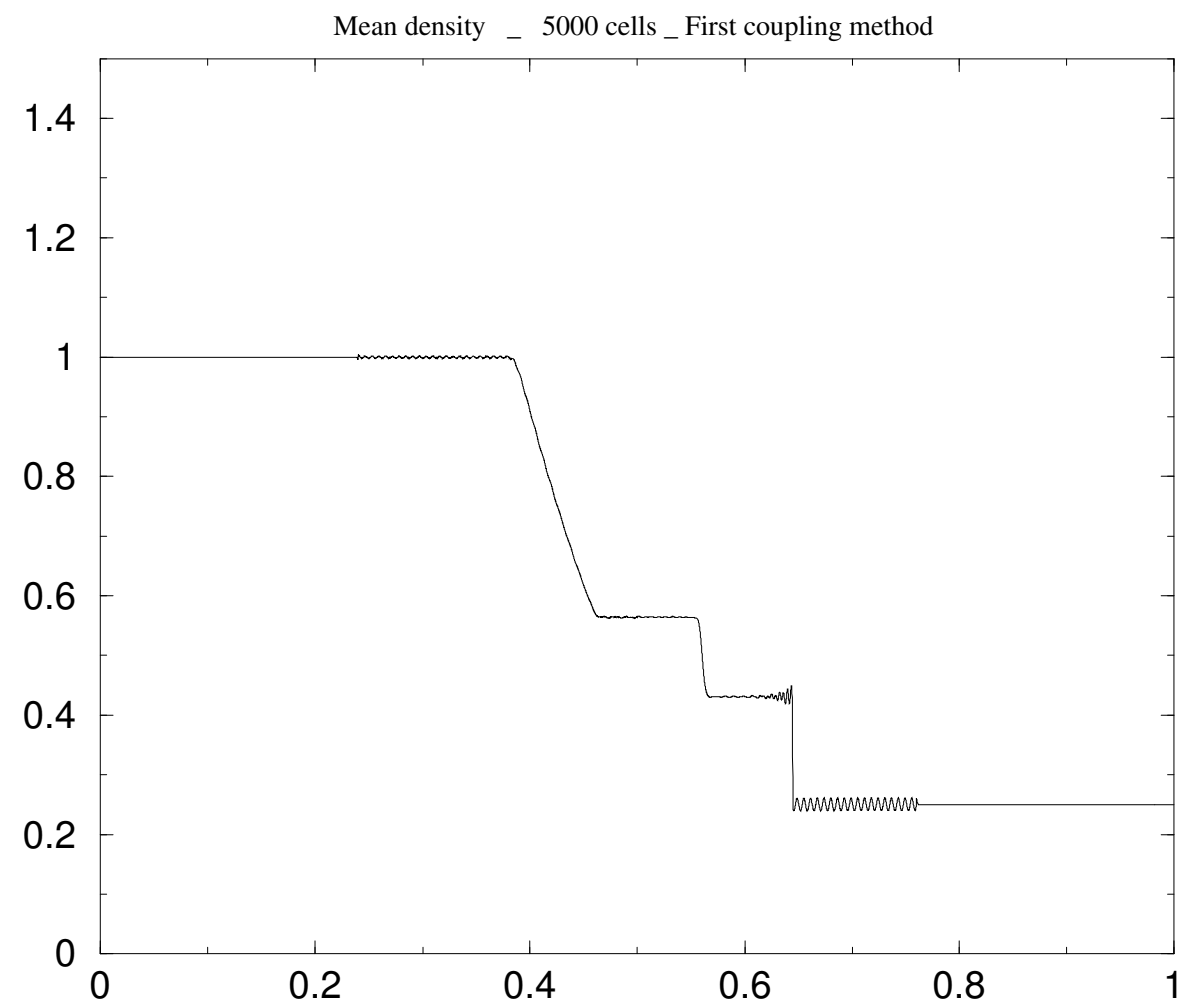

Figure 1. Mean density when solving a Riemann problem with sinusoidal signal

\section{Acknowledgments}

This work has been achieved while the author was on stay at CMI-LATP, UMR CNRS 6632, Université de Provence, Marseille, France.

\section{References}

${ }^{1}$ B. Audebert , Méthodes numériques pour les modeles de turbulence compressible avec équations aux tensions de Reynolds, PhD thesis, Université Pierre et Marie Curie, Paris VI, Paris, France, 2006,to appear.

${ }^{2}$ B. Audebert And F. Coquel, The Riemann problem for a class of Reynolds stress models, AIAA paper 2005-5103, 2005 ,

${ }^{3} \mathrm{C}$. Berthon, Contribution à l'analyse numérique des équations de Navier-Stokes à deux entropies spécifiques. Application à la turbulence compressible, Université Pierre et Marie Curie, Paris VI, Paris, France, 1999, january 22.

${ }^{4}$ C. Berthon, F. Coquel, J.M. Hérard, and M. Uhlmann, An approximate solution of the Riemann problem for a realisable second moment turbulent closure, Shock Waves, 2002, vol. 11-4, pp. 245-269.

${ }^{5}$ T. Brun, J.M. Hérard, D. Jeandel, and M. Uhlmann, An approximate Riemann solver for a class of realisable second-moment closures, Int. J. for Comp. Fluid Dyn., 2000, vol. 13-3, pp. 223-249.

${ }^{6}$ T. Buffard, T. Gallouët And J.M. HÉrard, A naive Riemann solver to compute a non conservative hyperbolic system, Int. Series in Numerical Mathematics, 1999, vol. 129, pp.129 -138.

${ }^{7}$ T. Buffard, T. GallouËt and J.M. HÉrard, A sequel to a rough Godunov scheme. Application to real gas flows, Computers and Fluids, 2000, vol. 29-7, pp. 813-847.

${ }^{8}$ S. Chibbaro, J.M. HÉrard and J.P. Minier, A novel hybrid moments/ moments-PDF method for turbulent two-phase flows, internal report, 2006. 
Riemann problem using imposed Reynolds stress profile $(\mathrm{CFL}=0.5)$

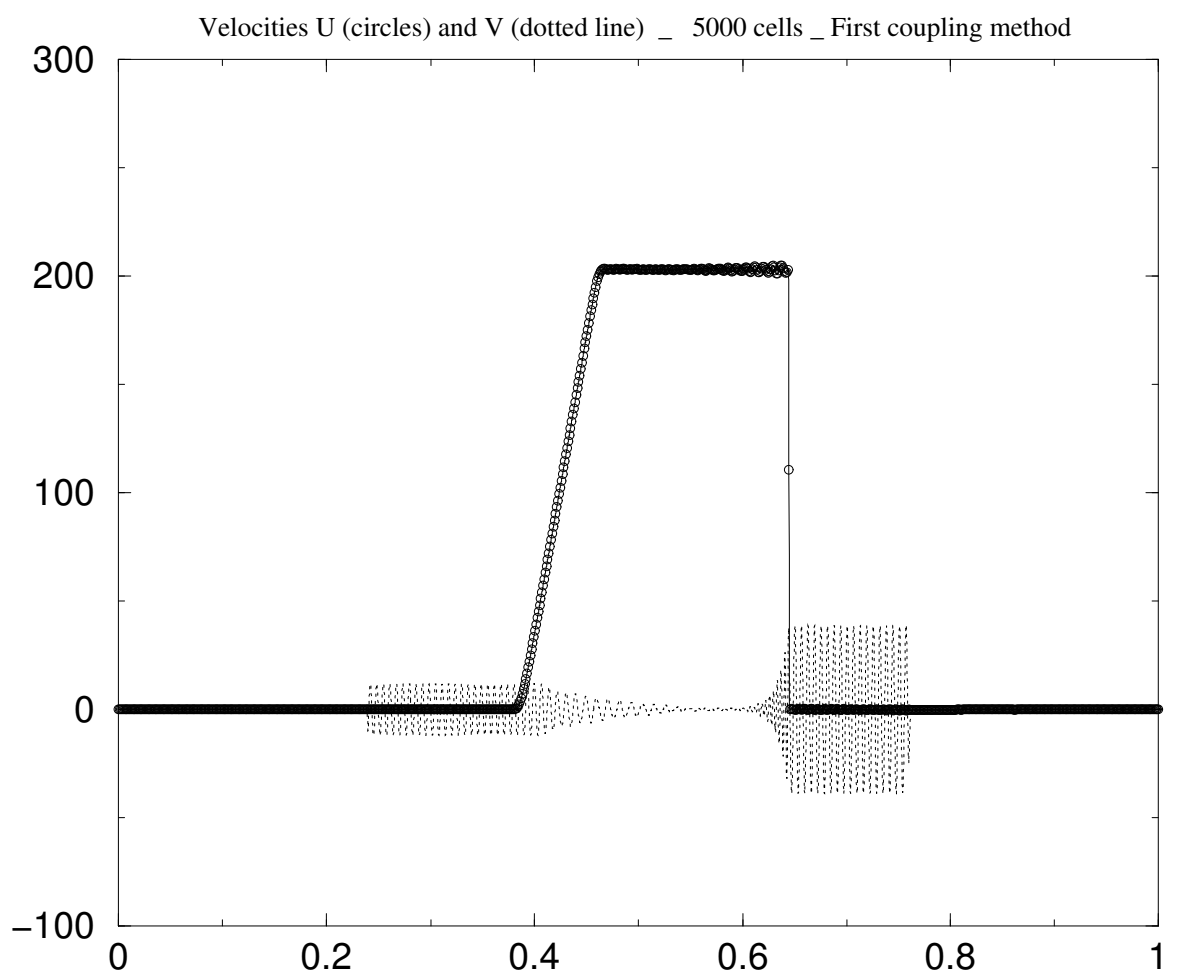

Figure 2. Mean velocities when solving a Riemann problem with sinusoidal signal

${ }^{9}$ F. Coquel and B. Perthame, Relaxation of energy and approximate Riemann solvers for general pressure laws in fluid dynamics, SIAM J. of Num. Anal., 1998, vol. 35, pp. 2223-2249.

${ }^{10}$ E. Godlewski And P.A. Raviart, Numerical approximation of hyperbolic systems of conservation laws, Springer Verlag, 1996.

${ }^{11}$ S.K. Godunov, A difference method for numerical calculation of discontinuous equations of hydrodynamics, Mat. Sb., 1959, pp. 271-300. In Russian.

12 J.M. HÉRARD, Basic analysis of some second moment closures. Part I : incompressible isothermal turbulent flows, Theor. Comp. Fluid Dyn.,1994, vol. 6(4), pp. 213-233.

${ }^{13}$ J.M. HÉRARD, Modeles au second ordre realisables non degeneres pour les ecoulements turbulents incompressibles, Comptes Rendus Académie des Sciences de Paris, 1996, vol. 322, pp. 371-377.

${ }^{14}$ J.M. HÉRARD, An analysis of second moment closures for incompressible turbulent flows with buoyant effects, submitted, 2005.

${ }^{15}$ T.P. Liu, G.Q. Chen And C.D. Levermore, Hyperbolic conservation laws with stiff relaxation terms and entropy, Comm. Pure Appl. Math., 1993, vol. 47-6 , pp.755-781.

${ }^{16}$ J.L. LumLEY, Computational modelling of turbulent flows, Advances in Applied Mechanics, 1978, vol. 18, pp.123-176.

${ }^{17}$ J.P. Minier, Probabilistic approach to turbulent two-phase flows modelling and simulation: theoretical and numerical issues, Monte Carlo Methods and Applications, 2001, vol. 7, pp. 295-310.

${ }^{18}$ J.P. Minier and E. Peirano, The PDF approach to turbulent polydispersed two-phase flows, Physics reports, 2001, vol. 352, pp. 1-214.

${ }^{19}$ M. Muradoglu, S.B. Pope, P. Jenny and D.A. Caughey, A consistent hybrid Finite Volume / particle method for the PDF equations of turbulent reactive flows, J. of Comp. Physics, 1999, vol. 154, p. 342.

${ }^{20}$ M. Muradoglu, S.B. Pope and D.A. Caughey, The hybrid method for the PDF equations of turbulent reactive flows: consistency conditions and correction algorithms, J. of Comp. Physics, 2001, vol. 172, pp. 841-878.

${ }^{21}$ S.B. Pope, Lagrangian PDF methods for turbulent flows, Ann. Rev. of Fluid Mechanics, 1994, vol. 26 , pp. 23.

${ }^{22}$ S.B. Pope, On the relationship between stochastic Lagrangian models of turbulence and second moment closures, Physics of Fluids, 1994, vol. 6, pp. 973-985.

${ }^{23}$ U. Schumann, Realisability of Reynolds stress turbulent models, Physics of Fluids, 1977, vol. 20, pp. 1033-1037.

${ }^{24}$ J. SMOLLER, Shock waves and reaction diffusion equations, Springer Verlag, 1983.

${ }^{25}$ C.G. Speziale, Invariance of turbulent closures revisited, Physics of Fluids, 1978, vol. 22, pp. 721-725. 
Riemann problem using imposed Reynolds stress profile ( $\mathrm{CFL}=0.5$ )

Mean pressure _ 500 cells and 5000 cells (dotted line)_First coupling method

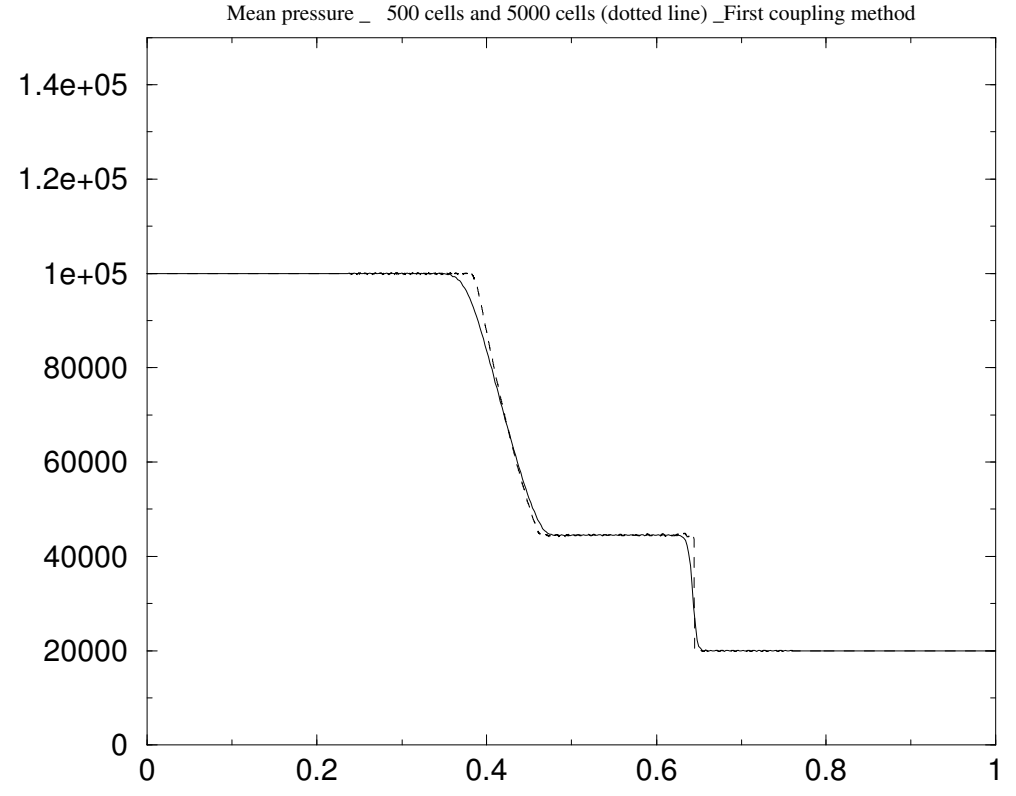

Figure 3. Effect of mesh refinement - Mean pressure when solving a Riemann problem with sinusoidal signal

Riemann problem using random data for Reynolds stress profiles

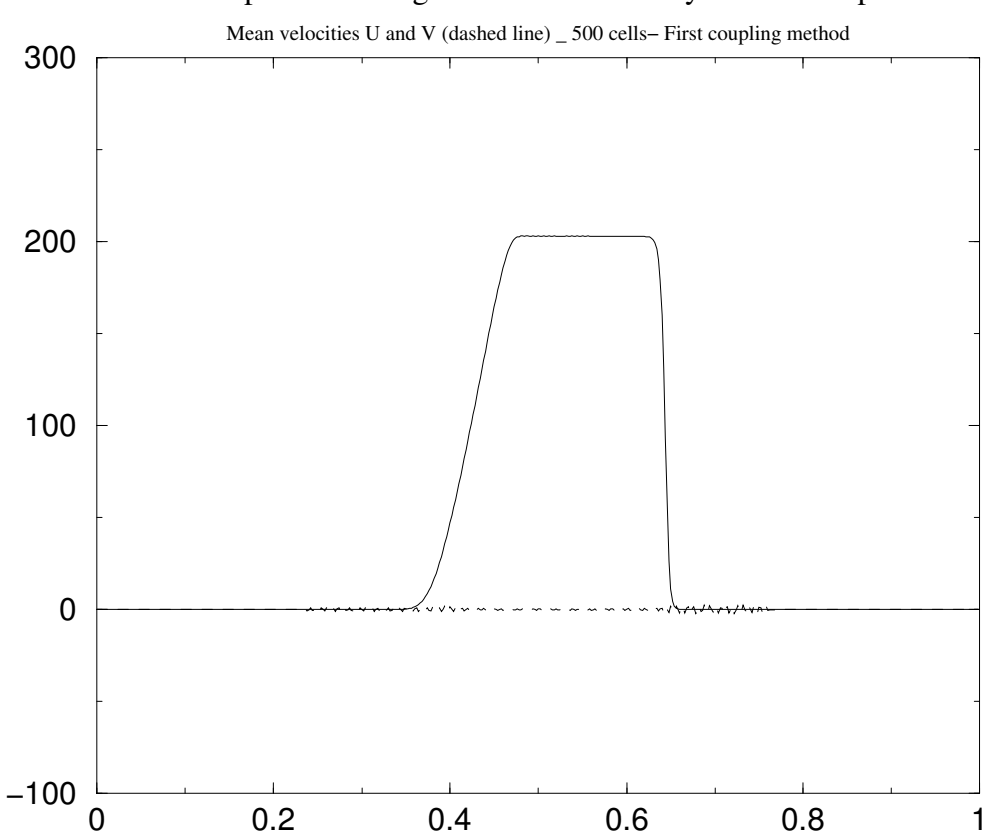

Figure 4. Mean velocities when solving a Riemann problem with random data 


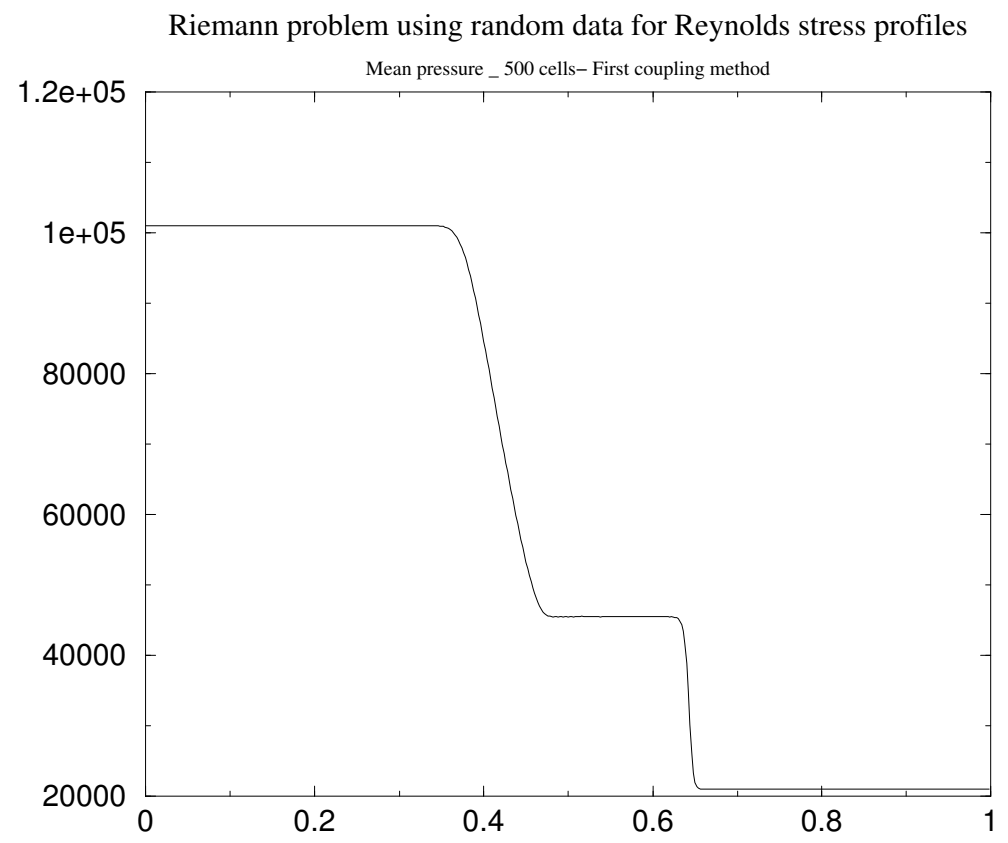

Figure 5. Mean pressure when solving a Riemann problem with random data

Riemann problem using random data for Reynolds stress profiles

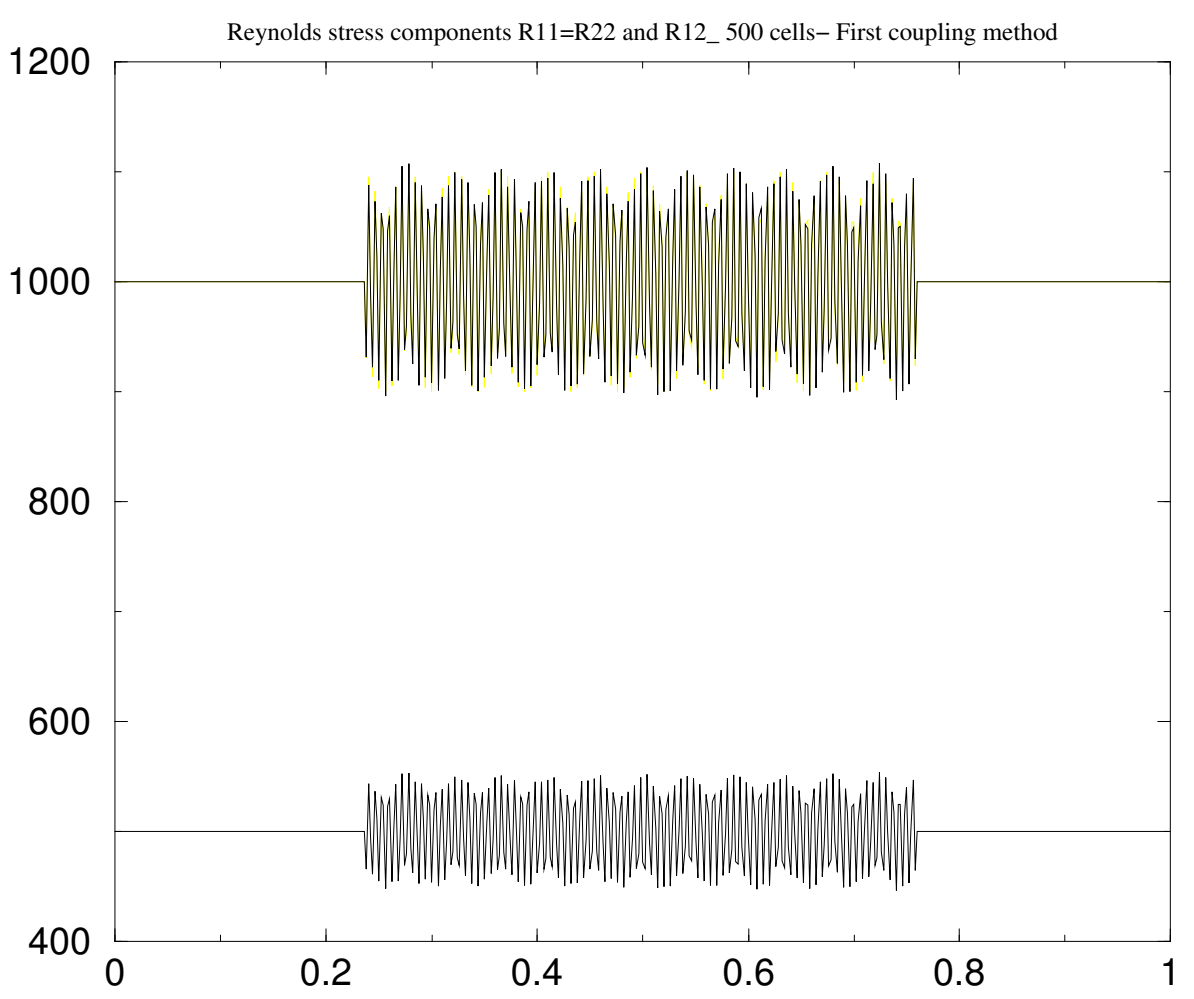

Figure 6. Reynolds stress components: random data

10 of 10 